\title{
Increase of the lethal activity of Cytotoxic T cells and Natural killer cells as a result of combination between Con-A and IL-2 in the initial stages from the treatment of lung cancer patients
}

\author{
mohmmed salem ${ }^{1}$, Nehal Elmashad ${ }^{2}$, Ismail Hassan ${ }^{3}$, and Mohamed Attia ${ }^{4}$ \\ ${ }^{1}$ Tanta University Faculty of Science \\ ${ }^{2}$ Tanta University Faculty of Medicine \\ ${ }^{3}$ Al-Azhar University Faculty of Science \\ ${ }^{4}$ Faculty of Medicine
}

April 28, 2020

\begin{abstract}
Cytotoxic $\mathrm{T}$ cells are one of the most specialized immune cells to defend the body against foreign invaders in the best scenario, but failure in the function of these cells, leading to decrease defense of the body against cancer. We aims to address the functionality of cytotoxic T lymphocytes (CTLs), Natural killer (NK) and Natural killer T-cells (NKT) cells in lung cancer patient's pre and post-stimulation of these cells in-vitro using laboratory activation protocol. Five healthy persons as volunteers and fifteen lung cancer patients were subjected to this study. The count of CTLs, NK and NKT cells and the expression of their intracellular granzyme B (GzB) pre and post-stimulation for $72 \mathrm{hrs}$ in-vitro was determined. The plasma level of cytokines and chemokines was correlated with the patient's prognosis. Data showed that after culture, there were highly significantly increased in the fold change of the percentage of GzB expression on CD3, CD4, CD8 and NKCD8 T cells in lung cancer patients before induction of chemotherapy when compared to healthy control and other lung cancer patients. The level of Proinflammatory cytokines in patients before and during induction of chemotherapy showed IL-1 and CCL-2 in a 13000, 250 fold increase respectively while it decreases in patients after induction of chemotherapy compared with the control group. Patients before the induction of chemotherapy have the ability to improve their CTL and NK functionality under activation conditions. The use of immune activation mechanisms is needed before the induction of chemotherapy.
\end{abstract}

\section{Introduction}

The progression of lung cancer is known to be linked with an overall weakened antitumor immunity[1] . Therefore, novel approaches for its treatment have been developed with the key objective of inhibiting this attenuated immune mechanism on one hand and activating self-defense [2] . Tremendous progress has been made to unveil the mechanism of the immune cell defense against cancer cells, presenting new and promising therapies based on new immunotherapy, represented in Immune checkpoint inhibitors, Antigenspecific vaccines and Adoptive $\mathrm{T}$ cell transfer (CAR T-cell), which augment the immune system in targeting cancer cells [3] . These promising methods can potentially provide long term cancer remission without causing the same side effects evident when using chemotherapeutic agents and radiation. In addition, they hold great promise in improving the prognostic outcomes of lung cancer patients, overcoming the hurdle of the poor overall 5-year survival rate barely ranging between 5-15\% [4].

Lung cancer cells elicit an immune response that recruits cytolytic immune cells including cytotoxic $\mathrm{T}$ cells $\left(\mathrm{CD}^{+}\right)$and natural killer cells (NK). These cells are able to remove harmful cells via the release of the cytolytic content of their granules such as perforin(Pr), granzymes (Gzs) and interferon-gamma (IFN- $\gamma$ ) into 
the cytosol of targeted cells ultimately leading to decomposing these cells[5] . Granzymes have the ability to target cells promoting downstream caspase activation and cell death. However, out of this serine protease granzyme family, Granzyme B (GzB) poses the major constituent excreted by CD8 ${ }^{+}$and NK and holds the strongest apoptotic activity of all Gzs [6] . Therefore, the ability of cancer cells to resist these cytolytic mediators plays a vital role in their continued existence and expansion.

Addressing the functionality of immune cells is of major importance to enable the evolvement of more effective immunotherapeutic approaches. Previous studies have demonstrated that lung cancer is associated with a locally decreased expression of $\mathrm{GzB}, \mathrm{Pr}$ and $\mathrm{IFN}-\gamma$ by the tumor-infiltrating $\mathrm{CD}^{+} \mathrm{T}$ cells, NK and NK T like cells in the tumor microenvironment by the effect of lung cancer mediators like prostaglandin E2 (PGE2), transforming growth factor (TGF)- $\beta$ ) which have a relationship with assisting tumor cell proliferation, anti-apoptotic properties, angiogenesis and chemotherapeutic resistance $[\mathbf{7}, \mathbf{8}]$. These alterations within the tumor microenvironment were found to impair the functionality of these cells and reduce the effectiveness of the adaptive immune responses against cancer cells. Accordingly, the hypothesis that this dysfunction extends beyond the confined boundaries of the tumor microenvironment has been validated [9] . we conducted the previous study concluded that the numbers of CTLs and NK cells and their expression of $\mathrm{GzB}$ were reduced in newly diagnosed lung cancer patients[10] .

In This study, we aim to address the number and functionality of CD4, CD8, NK, NKCD4 and NKCD8T cell in lung cancer patients before, during and after induction of chemotherapy and their intracellular expressions of $\mathrm{GzB}$ versus the inflammatory and prognosis cytokines and interleukins correlates it with the clinical response of patients in order to activation conditions with Concanavalin A (Con A) and Interleukin-2 (IL-2) in certain settings of culture in-vitro .

Material and methods:

\section{Subjects populations}

In this study, 15 patients of lung cancer and 5 healthy individuals were included. Blood samples were obtained from volunteers and lung cancer patients under treatment at Oncology Department, Tanta University Hospital. All patients are given informed consent under a protocol approved by the Faculty of Medicine Ethical Committee Review Board, Tanta University. Patients were diagnosed according to world health organization (WHO) criteria based on TNM classification[11] . Classification of subjects was performed after the detection of inclusion criteria and exclusion criteria before, during and after induction of chemotherapy.

\section{Chemical and reagents}

Lymphocyte separation medium (Ficoll paqué ${ }^{\mathrm{TM}}$ ) was purchased from Lonza (Basel, Switzerland). RPMI 1640 was purchased from Biochrom (Berlin, Germany) human serum was purchased from Bio Whittaker ( Walkersville, MD), L-glutamine was purchased from Life Technologies (Paisley, Scotland), penicillin, streptomycin, Concanavalin A (Con A) and Brefeldin A was purchased from Sigma (Sigma Chemical Co., Munich, Germany), Interleukin-2 (IL-2) were obtained from R\&D Systems (Minneapolis, Minnesota, USA). Phosphate buffer saline ( PBS) was obtained from (Verviers, Belgium). CD3 (perCP.Cy5*5), CD3 (APC) clone (RXB-14/IG), CD4 (Allophycocyanin APC-A), clone ( SK3) CD56 (FITC), granzyme B (GzB) Phycoerythrin (PE) clone(RXB-14/IG9), FACS Perm were purchased from (BD Biosciences (BD), San Jose, CA, USA). Sheath Fluid was purchased from (Luminex Corp, Austin, TX, USA).

\section{Preparation of samples}

\section{PBMCs separation}

Blood collection was on $\mathrm{K}_{2}$ EDTA tubes for plasma separation, PBMCs were separated using Ficoll paque ${ }^{\mathrm{TM}}$ technique in duplicate using break off centrifugation, twice washing using cold PBS, counting using hymocytometer to check the viability using trypan blue and to determine the number of PBMCs in the sample and the actual number of cultured cells[12] .

\section{Surface and intracellular staining of PBMCs before and after culture}


After PBMCs separation using Ficoll, 200,000 cells were separated immediately after counting for surface staining of anti-CD3 (perCP.Cy5.5), anti-CD4 (APC-A) and anti- CD56 (FITC) and intracellular for GzB by using anti-GzB (PE) [13].

\section{PBMCs culture}

$\left(1 \times 10^{6} / \mathrm{ml}\right)$ cells of PBMCs were cultured, using complete RPMI 1640 supplemented with $2 \%$ human serum FPS, $2 \mathrm{~m}$ M L-glutamine, 50 units $/ \mathrm{ml}$ penicillin, and $50 \mathrm{ng} / \mathrm{ml}$ streptomycin in the presence of $5 \mu \mathrm{g} / \mathrm{ml}$ concanavalin A (Con A) and 50ng/ml interleukin-2 (IL-2). Then cultured cells were incubated at $\mathrm{CO}_{2}$ incubator at $37^{\circ} \mathrm{C}$ in atmospheric pressure of $5 \% \mathrm{CO}_{2}$ for $72 \mathrm{hrs}$. Brefeldin A was added to the cultured cells 3 hours before harvesting [14].

\section{Measurement of plasma cytokines \& chemokine's}

Luminex 200 xMAP Technology (Luminex Corp, Austin, TX) was used to measure plasma chemokine's, C-C motif ligand2 (CCL2), C-C motif ligand4 (CCL4), C-C motif chemokine 11 (CCL11), C-X-C motif chemokine ligand8(CXCL8), C-X-C motif chemokine ligand 10 (CXCL10), interleukin-1 (IL-1), Interleukin-5 (IL-5) and Interleukin-6 (IL-6) in plasma. Briefly, plasma was diluted to two folds, then incubated with specific label beads (Molecular Probes; Life Technologies, Carlsbad, CA) coated with antibodies, after several washes and incubation periods, the previous beads were detected with Luminex system (Luminex Corp, Austin, Tex, USA). Data were obtained and calculated using a 5-parametric curve fit using xPONENTß, version 4.03 in a blinded fashion with measurement performed with the FlexMAP3D system (Luminex Corp, Austin, Tex, USA)[15] .

\section{Statistical analysis}

Data were analyzed using SPSS software version 22 and Mann-Whitney or one-way analysis of variance (a nova) with post-hoc for non-parametric analyses. Correlations were performed using Spearman's rank test. Analyses were performed using SPSS software. p values $<0.05$ were considered significant.

\section{Results}

\section{Clinical characteristics of lung cancer patients}

Samples of blood were collected from 15 lung cancer patients 13 male and 2 females before during and after induction of chemotherapy, patients were in the median age of $55.52 \pm 4.81$ years with a long history of smoking (30 to 40 years). Patients were diagnosed with NSCLC (poorly differentiated Adenocarcinoma). To correlate between clinical response and functionality of T, NK and NKT cells, data from patients were analyzed retrospectively, patients demographics have been described intable1 .

Count of T, NK and NKT cell population lung cancer patients in compared to healthy control before and after $72 \mathrm{hrs}$ of culture with Con-A and IL-2

Flow cytometry analysis to note the count of the CD3, CD4, CD8, NK, NKT, NKCD4, and NKCD8 cell populations were conducted. Before culture, the count of all cell populations noted to be decreased in all groups of lung cancer patients when compared to healthy persons who show an increased count for cell populations Fig 1B.

However, after culture, the percentages of CD3, CD4, CD8, NK, NKT, NKCD4, and NKCD8 cells were shown to be increased slightly in all groups of lung cancer patients especially patients before induction of chemotherapy when compared with CTRL. On the other hand, the overall NK percentages were not significantly affected by culturing and exhibited minimal changes represented in a slight increase of their population in the group of patients before chemotherapy and a slight decrease in the groups of patients during and after chemotherapy. Meanwhile, the NKT subpopulation of cells also showed non-significant changes in all groups except the group of patients following treatment with chemotherapy, where it showed a marked reduction in its count after culturing was performed. As for the levels of NKCD4 cells, a notable increase was observed in both the groups of before and during chemotherapy, while decreasing in the patient group after 
chemotherapy. Finally, the NKCD8 cell population displayed a decrease in patients prior to the induction of chemotherapy as well as a marked decrease after induction of chemotherapy Fig 1C.

GzB expression on T, NK and NKT cell in lung cancer patients and healthy control before and after $72 \mathrm{hrs}$ of culture with Con-A and IL-2

GzB expression was investigated in CD3, CD4, CD8, NK, NKT, NKCD4, and NKCD8 cells also by means of flow cytometry before and after culture with of IL-2 and Con-A. This also was carried out on 4 different groups: healthy controls and lung cancer patients before, during and after induction of chemotherapy.

Before culture, it was clear that CD8 and NK populations were the main secretary cells of GzB, with the remaining cell populations exhibiting minimal expression levels of it. Patients after induction of chemotherapy show a significant increase in the secretion of $\mathrm{GzB}$ when compared with healthy control and patients before and during induction of chemotherapy and this was generally applied for all cell populations.

After culturing, results demonstrated an increase of GzB expression in all populations of cells in most groups. Expression of $\mathrm{GzB}$ on $\mathrm{CD} 3$ and $\mathrm{CD} 4$ cells showed a notable increase in all groups with a less marked increase in the group of patients after chemotherapy. As of CD8 cells, results highlighted a striking increase in GzB expression levels before and during induction of chemotherapy as an effect of culturing in the activating media. Meanwhile, it demonstrated a moderate increase in the CTRL and the patients after the chemotherapy group. As for the expression of GzB on NK cells, percentages were increased significantly in CTRL only, while, no changes were detected in lung cancer patients before, during or after induction of chemotherapy after culture.

Meanwhile, GzB expression on NKT cells was increased significantly in CTRL, while displaying no changes in lung cancer patients before, during and after induction of chemotherapy. As of NKCD4 secretion, a significant increase was observed in CTRL, while a significant decrease was evident in lung cancer patients after induction of chemotherapy. Finally, NKCD8 was found to be considerably increased in CTRL, while no changes were noted in lung cancer patients before, during and after induction of chemotherapy fig 2.

Analysis of the fold change of T, NK and NKT cell count in lung cancer patients compared to CTRL from before to after culture

The fold change in the CD3, CD4, CD8, and NKT populations showed greatly increased in a patient group before chemotherapy when compared to CTRL while showing a less significant increase in the during and after induction of chemotherapy patient groups. Then again, NK and NKCD8 fold change percentages were not markedly changed in lung cancer patients before and during induction of chemotherapy compared to CTRL, yet they displayed a significant decrease after induction of chemotherapy. Moreover, NKCD4 cells showed a considerable increase in the fold change percentage in lung cancer patients before induction of chemotherapy and in contrast, a significant decrease was noted in patients after induction of chemotherapy when compared to the CTRL group fig $\mathbf{3 A}$.

Analysis of the fold change of granzyme B expression on T, NK and NKT cells in lung cancer patients compared to CTRL from before to after culture

when analyzing results in terms of fold changes of GzB expression on CD3, CD4, CD8, NK, NKT, NKCD4 and NKCD8 cells in lung cancer patients and CTRL before culture with after culture, all cell populations except the NK cells showed a marked increase in their expression levels of GzB upon activation in the newly diagnosed non-treated lung cancer group of patients. Similarly, these CD3, CD4, CD8, NKT, NKCD4 and NKCD8 cells all demonstrated a significant fold reduction during and after induction of chemotherapy when compared to CTRL. However, NK cell population where shown to be almost indifferent to the activation conditions, exhibiting no significant discrepancy in their fold change when compared to the CTRL fig 3B.

Analysis of cytokines, chemokine's and interleukins profiles of plasma of patients compared to CTRL 
Several cytokines and chemokines were measured from healthy, before, during and after induction of chemotherapy lung cancer patients, CXCL8 shows a highly significant increase in lung cancer patients before induction of chemotherapy while slightly increase were shown in lung cancer patients during and after induction of chemotherapy when compared to healthy CTRL. Interestingly, IL-6 and IL-1 and shows a highly significant increase in lung cancer patients before and during induction of chemotherapy (100 and 13000 fold increase respectively) and no significant change in patients after induction of chemotherapy when compared to CTRL. According to CCL2, there is a highly significant increase in lung cancer patients before (250 fold) during and after induction of chemotherapy when compared to CTRL. CXCL10 shows a highly significant increase in lung cancer patients before and during the induction of chemotherapy when compared to CTRL. On the other hand IL-5, CCL11 and CCL4 show no significant change in their percentages in any group compared to the CTRL group fig 4.

\section{Discussion}

Immune surveillance is the process in which precancerous and malignant cells are detected by the immune system as damaged cells and are consequently targeted for elimination. Cytotoxic T cells, NK and NK-like $\mathrm{T}$ cells are indispensable factors in the body's ongoing defense against these transformed tumor cells. This Cytotoxic function is a highly regulated, multi-factorial immune process that is carried out via using a granule exocytosis pathway to induce granzyme-dependent death in targeted cells. For this reason, in order for lung cancer cells to survive, they must hold certain properties that enable them to evade and resist these cytotoxic cell attacks. In this regard, previous studies have shown that malignant tumor cells are able to escape immune surveillance and thrive [16]. Lung cancer cells produce mediators such as prostaglandin E2 (PGE2) and transforming growth factor (TGF)- $\beta$ that further support tumor cell proliferation, anti-apoptotic properties and chemotherapeutic resistance [17] . Therefore, a broader understanding of the different immunosuppressive strategies which foster immune evasion, preventing host immune cells from carrying out an effective anti-tumor immune response is needed for the identification of new therapeutic strategies that can potentially improve treatment outcomes in lung cancer patients. The tumor microenvironment (TME) in lung cancer has been a subject of intensive and rigorous studies. The capacity of tumor cells to reshape the constitution and function of stromal and immune cells at the tumor site eventually creating an immunosuppressive milieu. Many studies have reported that this local impairment of immune cells renders them less tumoricidal and thereby supporting the progression of cancer. For example, intratumoral NK cells exhibited profound defects in their ability to produce interferon-gamma and activate degranulation[5]. In a similar manner, both CD4+ and CD8+ cells from lung cancer tissue demonstrated a significant decrease in intracellular levels of granzyme B compared to non-cancerous tissue [18] . On the other hand, cytokines and chemokines that promote angiogenesis, chemoresistance and activate immune suppressive cells (T-regs and MDSCs) have found to exhibit an alteration in their expression within the TME. Collectively, the critical role the TME plays in altering the biological behaviors of tumors has been well established. However, information regarding the systemic effect that tumor cells exert in lung cancer patients remains limited. As increasing evidence suggests that tumors are able to manipulate the systemic immune components, in our study we aimed to clarify the changes in the peripheral immune environment in terms of numbers and functionality of immune cells as well as cytokine levels present in peripheral blood of lung cancer patients[19] .

This study was conducted on 15 patients diagnosed with lung cancer at different stages before $(\mathrm{n}=5)$, during $(\mathrm{n}=5)$ and after $(\mathrm{n}=5)$ induction of chemotherapy as they were compared to healthy donors $(\mathrm{n}=5)$. All patients were in the age group ranging between 35 to 65 years and showed an obvious male predominance with a male: female ratio of 85:15. In addition, all male patients were found to be long-term positive smokers which confirms that smoking is a strong prognostic and predictive patient characteristic in lung cancer [20]

Selection of patients must be with a clear history of treatment with board spectrum antibiotics especially before chemotherapy treatment, the effect of inducing antibiotics make a loss of functionality by affecting the normal lymphocyte activation mechanisms of the immune response this was proved when using immune activation in-vitro or applying immunotherapy [21].

The aim was to determine the functionality of CD3, CD8, CD4, NK, NKT, NKCD4 and NKCD8 cells in lung 
cancer patients before, during and after induction of chemotherapy. We hypothesized that the peripheral immune components are affected to create a pro-tumor peripheral immune environment decreasing the number and functionality of immune cells. Therefore, we investigated the effect of culturing these immune cells inactivation conditions and comparing the results with our initial findings. As our previous study concluded that the numbers of circulating T, NK and NKT cells and their levels of GzB expression are decreased in lung cancer patients, The findings of these study suggest that lung cancer cells might decrease the proportion of peripheral of all the cell populations (CD3, CD8, CD4, NK, NKT, NKCD4 and NKCD8) cells by yet unidentified mechanisms. Notably, the present study also observed a decrease in the levels of GzB before and during induction of chemotherapy when compared to CTRL, while increasing to some extent after induction of chemotherapy. This combined deficiency in the count and functionality of the various lymphocyte subsets can be explained by the effect of the previously mentioned soluble factors produced from the tumor cells, which is in line with the previous studies of Zhao et al., who that these mediators are responsible for inhibition of CTL and NK function as secretors of GzB [10, 22] On the other hand, these findings may be also attributed to the effect of immune suppressive cells such as myeloid-derived suppressor cells (MDSCs) and regulatory T cells (Treg) which are correlated with the stages and cancer progression excreting their effect on immune cells $[\mathbf{2 3}, \mathbf{2 4}]$

However, after cultivating PBMCs in the stimulatory media, immense changes were observed in most populations of cells, reflecting an increase in the numbers of cells in the before, during and after chemotherapy stages. There was more evident in the early diagnosed lung cancer group of patients before induction of chemotherapy and in the CD3, CD8 and NKCD4 cells in specific. Likewise, the fold change in the expression of GzB on CD3, CD8, CD4 and NKT cells after culture were recorded to be significantly increased in lung cancer patients before induction of chemotherapy with clearly elevated percentages when compared to CTRL and other lung cancer patients. This can be explained by the effect of adding Con-A and IL-2 to the culturing media. Con-A is known for its effect as a stimulator of lymphocyte blastogenesis and mitosis, it is also known to induce the uptake of nucleotides, sugars, amino acids [25] . As of IL-2, it has been considered to be a key growth for antigen-activated $\mathrm{T}$ lymphocytes since it also maintains self-tolerance and causes a massive expansion of $\mathrm{CD} 8^{+}$cells [26] . Extensive studies on IL-2 have led to the clinical utilization of this molecule in patients with advanced cancer however this was limited due to the significant systemic side effects associated with its use. For this reason, attempting to overcome this obstacle without decreasing the antitumor potency current research is oriented towards developing combination regimens that have additive or synergistic antitumor effects with fewer toxicities [27]. Our study demonstrated that the combined use of IL-2 and Con-A promoted synergistic in-vitro expansion and activation of peripheral immune cells, specifically $\mathrm{CD}^{+}$cells. Therefore, the combined use of IL-2 and Con-A should be considered a practical strategy for the generation of antitumoral responses in-vivo and for future clinical applications.

On the contrary, the NK population of cells was not affected by this stimulatory effect in terms of either numbers or functionality. This suggests that since NK cells haven't been activated compared to CTL, CTL are the main secretors of $\mathrm{GzB}$ and that they have the ability to be activated to express $\mathrm{GzB}$ in abundance. However, NK cells have been previously identified as the main secretors of IFN- $\gamma$ which may explain why their expression levels of $\mathrm{GzB}$ were not potently affected

\section{$[28]$.}

NKT profile shows slightly increase without the significance of GzB expression, this is reported by Wang et al. who reported that their function is affected by the release of soluble major histocompatibility complex (MHC) class I chain-related molecules by cancer cells impairing the lytic activity via down-regulation of the NKG2D receptor [29].

Circulating cytokines are closely associated with the immune status against many diseases including cancer. In the present study, we also observed the levels of several cytokines in our different groups of patients compared to healthy controls. As shown in our previous study, the levels of IL-1 and CCL-2 in early diagnosis patients showed a 14000, 250 fold increase respectively compared with the control group. This increase adds to the chain of evidence supporting that these cytokines promote tumor growth, chemo- 
resistance and help sustain an immunosuppressive milieu [30]. Additionally, we found that the levels of IL-6 where increased by 100 fold in lung cancer patients which is also in line with previous studies has shown it to be positively correlated with lymph node metastasis, distant metastasis and worse overall survival $[30,31]$. Furthermore, CXCL8 which promotes angiogenesis and tumor progression was increased by 30 folds in diagnosed lung cancer patients weighed against healthy controls. Whereas the proportions of the other 4 cytokines evaluated, including CXCL10, IL-5, CCL11 and CCL4 showed no significant difference between lung cancer patients and the control group [32]. The present study supports the hypothesis that the systemic cytokine cascade exists in lung cancer patients, reflecting the tumor stage and host immune status. Moreover, these changes in the level of cytokines may be of major importance as a diagnostic and prognostic tool in lung cancer and they can be considered as potential targets for immunotherapy.

In summary, these results confirm that the functionality of CTL and NK cells are impaired in cancer patients during and after induction of chemotherapy, while, patients before induction of chemotherapy have the ability to improve their CTL and NK functionality under activation conditions of combination between Con-A and IL-2.

\section{References}

1. Singh, K., et al., Mst1/2 kinases restrain transformation in a novel transgenic model of Ras driven non-small cell lung cancer. Oncogene, 2019: p. 1-13.

2. Raniszewska, A., et al., PD-L1 expression on lung cancer stem cells in metastatic lymph nodes aspirates. Stem Cell Reviews and Reports, 2019. 15 (2): p. 324-330.

3. Lara-Guerra, H. and J.A. Roth, Gene therapy for lung cancer.Critical Reviews in Oncogenesis, 2016. $21(1-2)$.

4. Aktas, O.N., et al., Role of natural killer cells in lung cancer. Journal of cancer research and clinical oncology, 2018.144 (6): p. 997-1003.

5. Degos, C., et al., Endometrial tumor microenvironment alters human NK cell recruitment, and resident NK cell phenotype and function. Frontiers in immunology, 2019. 10 : p. 877.

6. Li, Y., et al., M860, a Monoclonal Antibody against Human Lactoferrin, Enhances Tumoricidal Activity of Low Dosage Lactoferrin via Granzyme B Induction. Molecules, 2019. 24 (20): p. 3640.

7. Barrett, C.S., A.C. Millena, and S.A. Khan, TGF-beta Effects on Prostate Cancer Cell Migration and Invasion Require FosB. Prostate, 2017. 77 (1): p. 72-81.

8. Caramori, G., et al., Molecular links between COPD and lung cancer: new targets for drug discovery? Expert opinion on therapeutic targets, 2019. 23 (6): p. 539-553.

9. Chollat-Namy, M., et al., The pharmalogical reactivation of p53 function improves breast tumor cell lysis by granzyme B and NK cells through induction of autophagy. Cell death \& disease, 2019.10 (10): p. $1-20$.

10. Hassan, I.A., et al., Low production of granzyme B by Cytotoxic $T$ cells and Natural killer cells associated with the increased levels of cytokines and chemokine's in early diagnostic lung cancer patients. International Journal of Cancer and Biomedical Research, 2020.

11. Travis, W., et al., ES12. 05 Impact of STAS in Lung Cancer Staging. Journal of Thoracic Oncology, 2019. 14 (10): p. S45.

12. Seeligmüller, N., Faster Isolation of PBMC Using Ficoll-Paque. Plus in the Eppendorfß Centrifuge 5920 R . 2016, Application Note.

13. Adan, A., et al., Flow cytometry: basic principles and applications. Critical reviews in biotechnology, 2017.37 (2): p. 163-176.

14. Green, K.J., D.G. Rowbottom, and L.T. Mackinnon, Exercise and T-lymphocyte function: a comparison of proliferation in PBMC and NK cell-depleted PBMC culture. Journal of Applied Physiology, 2002.92 (6): p. 2390-2395.

15. Datta, S.C. and M.R. Opp, Lipopolysaccharide-induced increases in cytokines in discrete mouse brain 
regions are detectable using Luminex $x M A P(\mathbb{R}$ technology. Journal of neuroscience methods, 2008.175 (1): p. 119-124.

16. Lin, X.-R., et al., CIK cell-based delivery of recombinant adenovirus KGHV500 carrying the antip21Ras scFv gene enhances the anti-tumor effect and safety in lung cancer. Journal of cancer research and clinical oncology, 2019. 145 (5): p. 1123-1132.

17. Jiang, X., et al., Role of the tumor microenvironment in PD-L1/PD-1-mediated tumor immune escape. Molecular cancer, 2019.18 (1): p. 1-17.

18. Huang, J.Y., et al., Circulating markers of cellular immune activation in prediagnostic blood sample and lung cancer risk in the Lung Cancer Cohort Consortium (LC3). International journal of cancer, 2019.

19. Giraldo, N.A., et al., The clinical role of the TME in solid cancer. British journal of cancer, 2019. 120 (1): p. $45-53$.

20. Butler, S.J., et al., Prevalence of lung cancer in chronic obstructive pulmonary disease: A systematic review. Respiratory Medicine: X, 2019: p. 100003.

21. Yan, C., et al., Antibiotics and immunotherapy in gastrointestinal tumors: Friend or foe? World Journal of Clinical Cases, 2019. 7 (11): p. 1253.

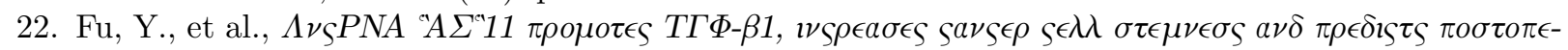

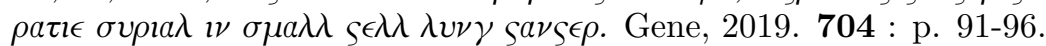

23. Diaz-Montero, C.M., et al., Increased circulating myeloid-derived suppressor cells correlate with clinical cancer stage, metastatic tumor burden, and doxorubicin-cyclophosphamide chemotherapy. Cancer immunology, immunotherapy, 2009. 58 (1): p. 49-59.

24. Alghamri, M.S., et al., Functional assay to assess T-cell inhibitory properties of myeloid derived suppressor cells (MDSCs) isolated from the tumor microenvironment of murine glioma models, inMethods in Enzymology . 2020, Elsevier. p. 215-228.

25. Cechova, K., et al., Up-regulation of mu-, delta- and kappa-opioid receptors in concanavalin A-stimulated rat spleen lymphocytes. J Neuroimmunol, 2018. 321 : p. 12-23.

26. Turajlic, S., et al., Tracking cancer evolution reveals constrained routes to metastases: TRACERx renal. Cell, 2018.173 (3): p. 581-594. e12.

27. De, U., et al., Plumbagin from a tropical pitcher plant (Nepenthes alata Blanco) induces apoptotic cell death via a p53-dependent pathway in MCF-7 human breast cancer cells. Food and chemical toxicology, 2019. 123 : p. 492-500.

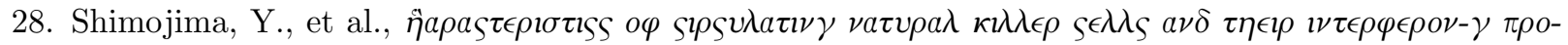

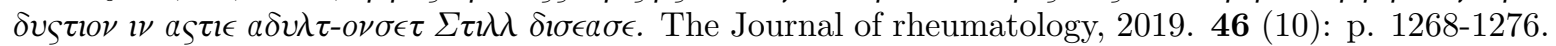

29. Wang, H., et al., Tumor-derived soluble MICs impair CD3+CD56+ NKT-like cell cytotoxicity in cancer patients. Immunology letters, 2008. 120 (1-2): p. 65-71.

30. Hu, Q., et al., Role of ALDH1A1 and HTRA2 expression in CCL2/CCR2-mediated breast cancer cell growth and invasion. Biology open, 2019. 8 (7): p. bio040873.

31. Saad, M.I., et al., ADAM17 selectively activates the IL-6 trans-signaling/ERK MAPK axis in KRASaddicted lung cancer. EMBO molecular medicine, 2019. 11 (4).

32. Donnenberg, A.D., J.D. Luketich, and V.S. Donnenberg, Secretome of pleural effusions associated with non-small cell lung cancer (NSCLC) and malignant mesothelioma: therapeutic implications. Oncotarget, 2019. 10 (60): p. 6456.

\section{Figure legends}

Figure1. (A) Gating strategy for Flowcytometry analysis of PBMCs from lung cancer patients before, during and after chemotherapy compared to healthy control before and After 72 hours of culture.(a) represents the all events from flowcytometry directed to the lymphocyte population. (b) represents the population of the total T cells CD3+, NK cells and NKT cells, (c) represents the population of CD8 cells and CD4. (d) represents the population of NKCD8 cells and NKCD4. (e) represents GzB expression on all mentioned populations. (B) Representative Flowcytometry analysis of CD3, CD8, CD4, NK, NKCD4 and NKCD8 cells from healthy control(a), lung cancer patients before(b), during(c) and after induction of chemother- 
apy (d)(before culture) (C) Representative Flowcytometry analysis of CD3, CD8, CD4, NK, NKCD4 and NKCD8 cells from healthy control(a), lung cancer patients before(b), during(c) and after induction of chemotherapy (d) (after culture).

Figure2. Levels of Intracellular GZB expression on CD3, CD8, CD4, NK, NKCD4 and NKCD8 cells from healthy control(a), lung cancer patients before(b), during(c) and after induction of chemotherapy (d) after culture in the presence of Con A $(5 \mathrm{ng} / \mathrm{ml})$ and IL-2 $(50 \mathrm{ng} / \mathrm{ml})$ and incubated for $72 \mathrm{hrs}$, then harvested and stained with the same protocol.

Figure3. (A) Statistical analysis of the fold change in the count of CD3 (a), CD4 (b), CD8 (c), NK(d) , NKT (e), NKCD4 (f) and NKCD8(g) cells from lung cancer patients before, during and after induction of chemotherapy (black bars), compared to healthy control (white bars) . (B) Statistical analysis of the fold change in the expression of GZB on CD3 (a), CD4 (b), CD8(c), NK (d) and NKT (e), NKCD4 (f)and NKCD8 (g) cells from lung cancer patients before, during and after induction of chemotherapy (black bars), compared to healthy control (white bars). Data were analyzed using SPSS software and Mann-Whitney or one-way analysis of variance (a nova) with post-hoc for non-parametric analyses, P-values $<0 * 05$ were considered significant (*and ** p [?] 0.05, p [?] 0.01).

Figure4. Statistical analysis of the expression of CXCL8, IL-6, IL-1, CCL2, CXCL10, IL-5, CCL11 and CCL4 in the plasma of lung cancer patients before, during and after induction of chemotherapy (black bars), compared to healthy control (white bars). Data were analyzed using SPSS software and Mann-Whitney or one-way analysis of variance (a nova) with post-hoc for non-parametric analyses, P-values $<0 * 05$ were considered significant $\left(*, * *\right.$ and ${ }^{* * *} \mathrm{p}$ [?] $0.05, \mathrm{p}$ [?] 0.01 and, p [?] 0.001).

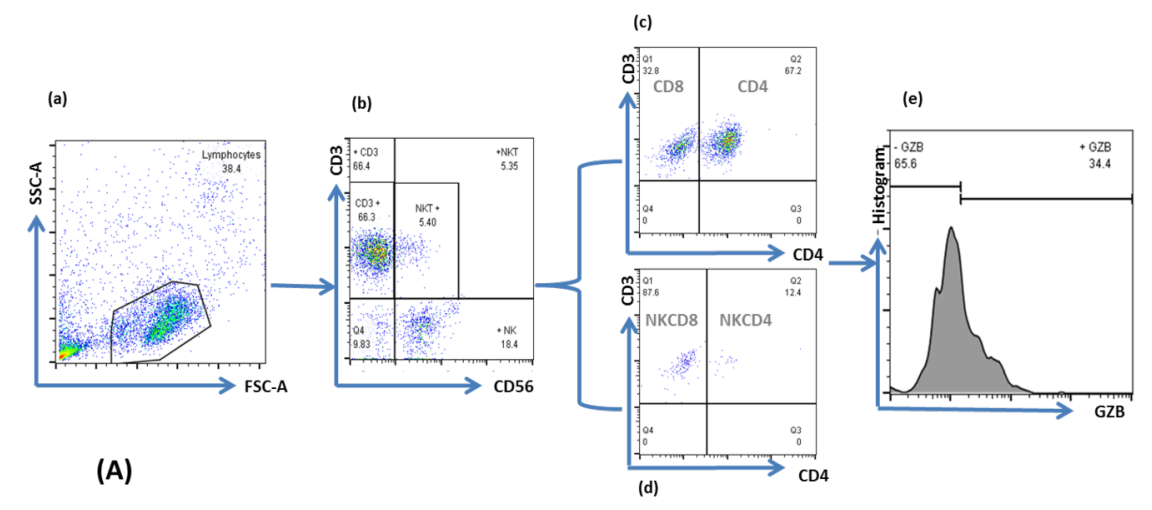

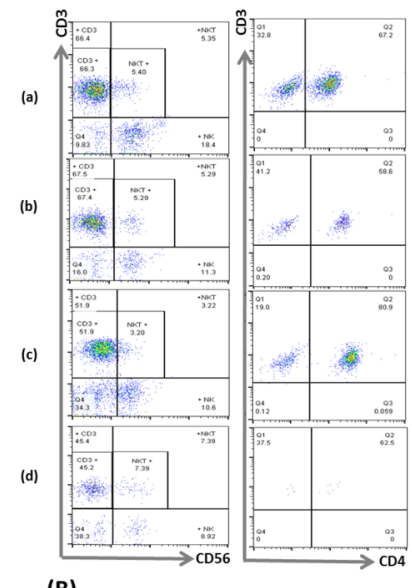

(B)

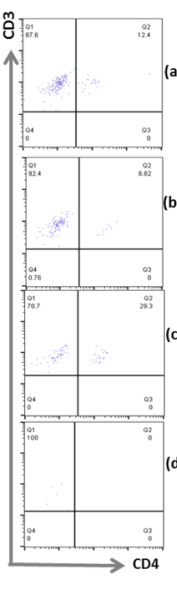

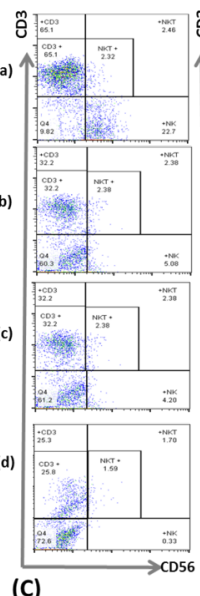

(C)
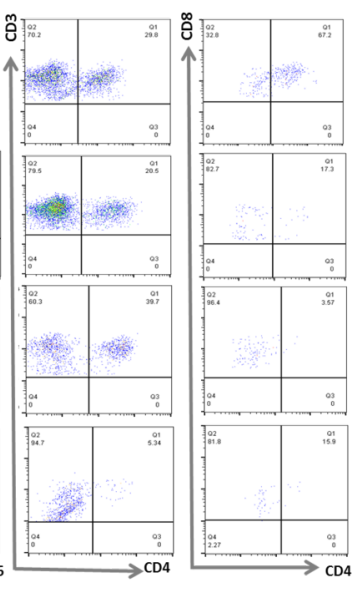
Fig.1
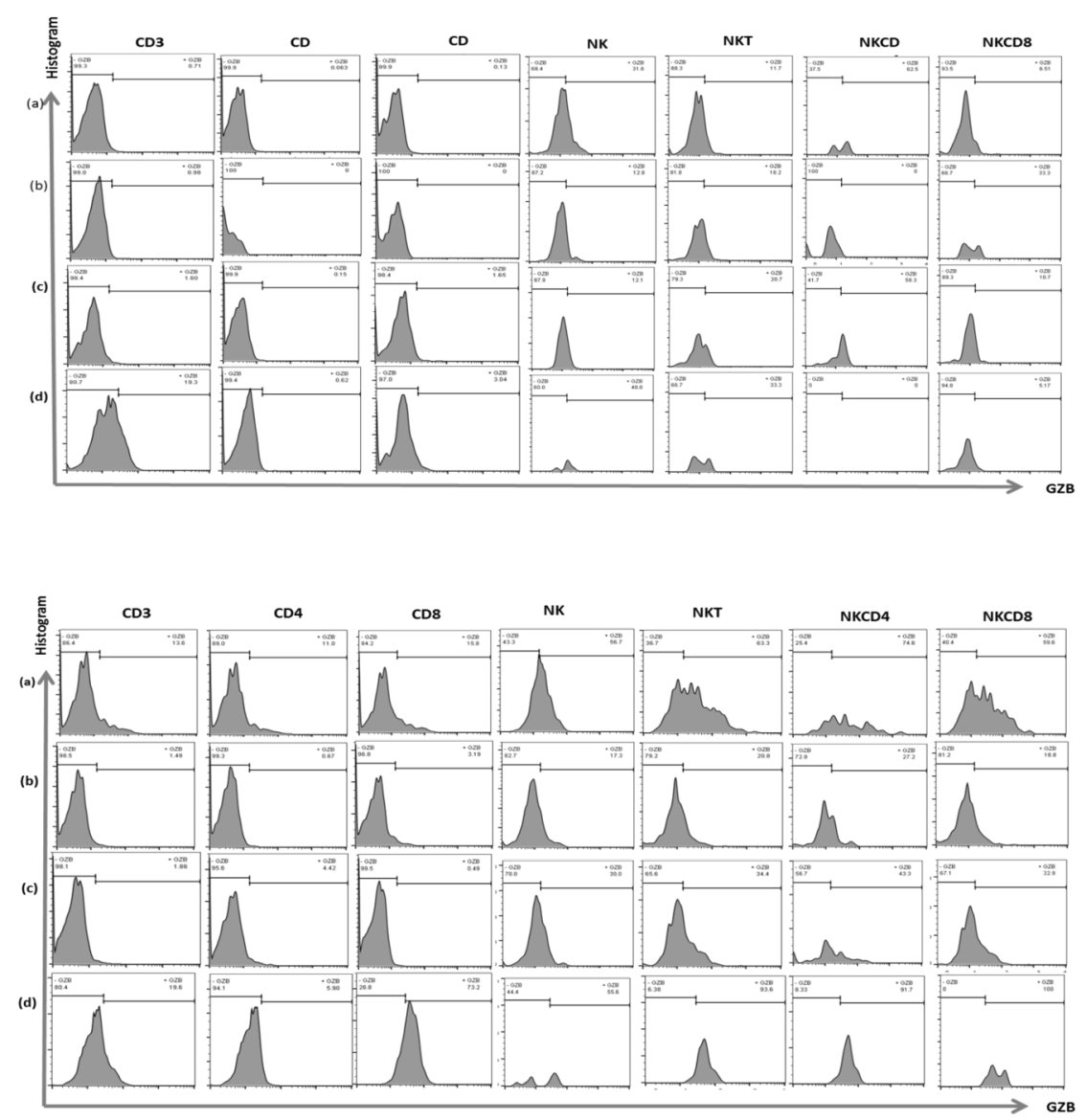

Fig.2

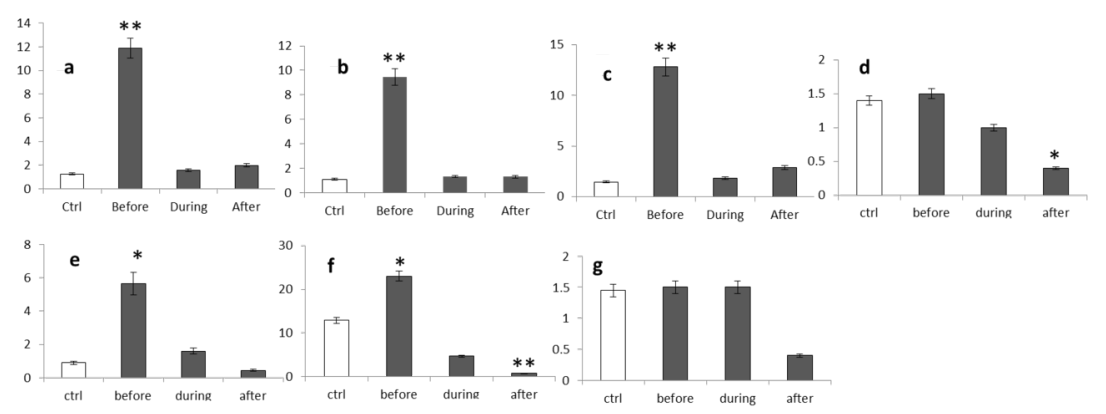

(A) 


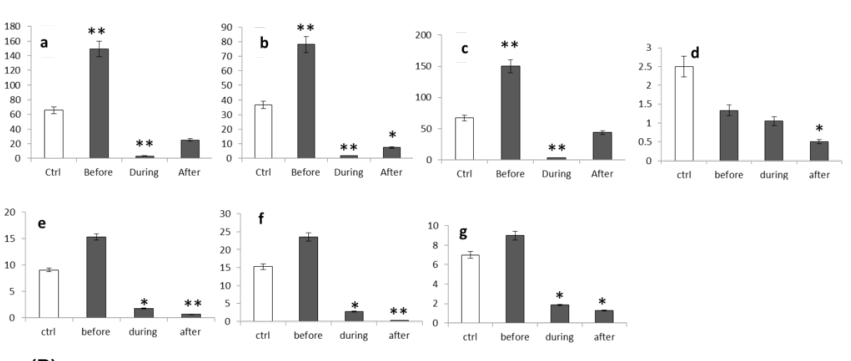

(B)

Fig.3
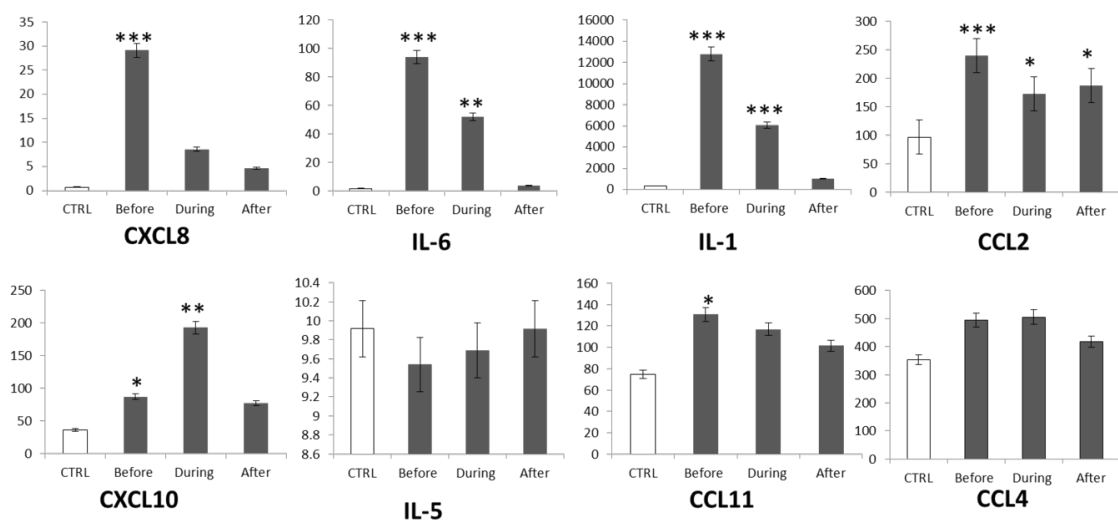

Fig.4

Table (1): patients demographics and different hematological and clinical parameters in patient and control groups

\begin{tabular}{|c|c|c|c|c|c|c|c|c|c|c|c|c|c|}
\hline \multirow[b]{2}{*}{ Sex\% } & \multicolumn{13}{|c|}{ Control Control Control PatientsPatientsPatientsPatientsPatientsPatientsPatientsPatientsPatientsPat } \\
\hline & & & & Before & Hefore & \%Before & \%Before & \%During & \%uring & \%urin & \$urin & $\%$ After\% & $\mathrm{Aft}$ \\
\hline Male & 80.0 & 80.0 & 80.0 & 90.0 & 90.0 & 90.0 & 90.0 & 90.0 & 90.0 & 90.0 & 90.0 & 100 & 100 \\
\hline Female & 20.0 & 20.0 & 20.0 & 10.0 & 10.0 & 10.0 & 10.0 & 10.0 & 10.0 & 10.0 & 10.0 & 0 & 0 \\
\hline Age & 33.0 & 33.0 & 33.0 & 35.0 & 35.0 & 35.0 & 35.0 & 35.0 & 35.0 & 35.0 & 35.0 & 35.0 & 35.0 \\
\hline Min. & - & - & - & - & - & - & - & - & - & - & - & - & - \\
\hline- & 40.0 & 40.0 & 40.0 & 75.0 & 75.0 & 75.0 & 75.0 & 75.0 & 75.0 & 75.0 & 75.0 & 75.0 & $75 .($ \\
\hline Max. & 36.32 & 36.32 & 36.32 & 55.52 & 55.52 & 55.52 & 55.52 & 55.52 & 55.52 & 55.52 & 55.52 & 55.52 & 55. \\
\hline Mean & \pm & \pm & \pm & \pm & \pm & \pm & \pm & \pm & \pm & \pm & \pm & \pm & \pm \\
\hline $\begin{array}{l} \pm \\
\text { SD. }\end{array}$ & 2.43 & 2.43 & 2.43 & 4.81 & 4.81 & 4.81 & 4.81 & 4.81 & 4.81 & 4.81 & 4.81 & 4.81 & SD. \\
\hline Smoker & & & & $>0.05$ & $>0.05$ & $>0.05$ & $>0.05$ & $>0.05$ & $>0.05$ & $>0.05$ & $>0.05$ & $>0.05$ & $>0$ \\
\hline Nil & 100.0 & 100.0 & 100.0 & 0.0 & 0.0 & 0.0 & 0.0 & 0.0 & 0.0 & 0.0 & 0.0 & 0.0 & 0.0 \\
\hline \multicolumn{14}{|l|}{$\begin{array}{l}\text { Ve- } \\
\mathrm{Ve}+\end{array}$} \\
\hline & 0.0 & 0.0 & 0.0 & 10.0 & 10.0 & 10.0 & 10.0 & 10.0 & 10.0 & 10.0 & 10.0 & 0.0 & 0.0 \\
\hline & 0.0 & 0.0 & 0.0 & 90.0 & 90.0 & 90.0 & 90.0 & 90.0 & 90.0 & 90.0 & 90.0 & 100.0 & 100 \\
\hline & & & & $<0.002$ & $*<0.00$ & $*<0.00$ & $*<0.00$ & $*<0.00$ & $*<0.002$ & $*<0.00$ & $*<0.00$ & $*<0.001$ & \\
\hline
\end{tabular}




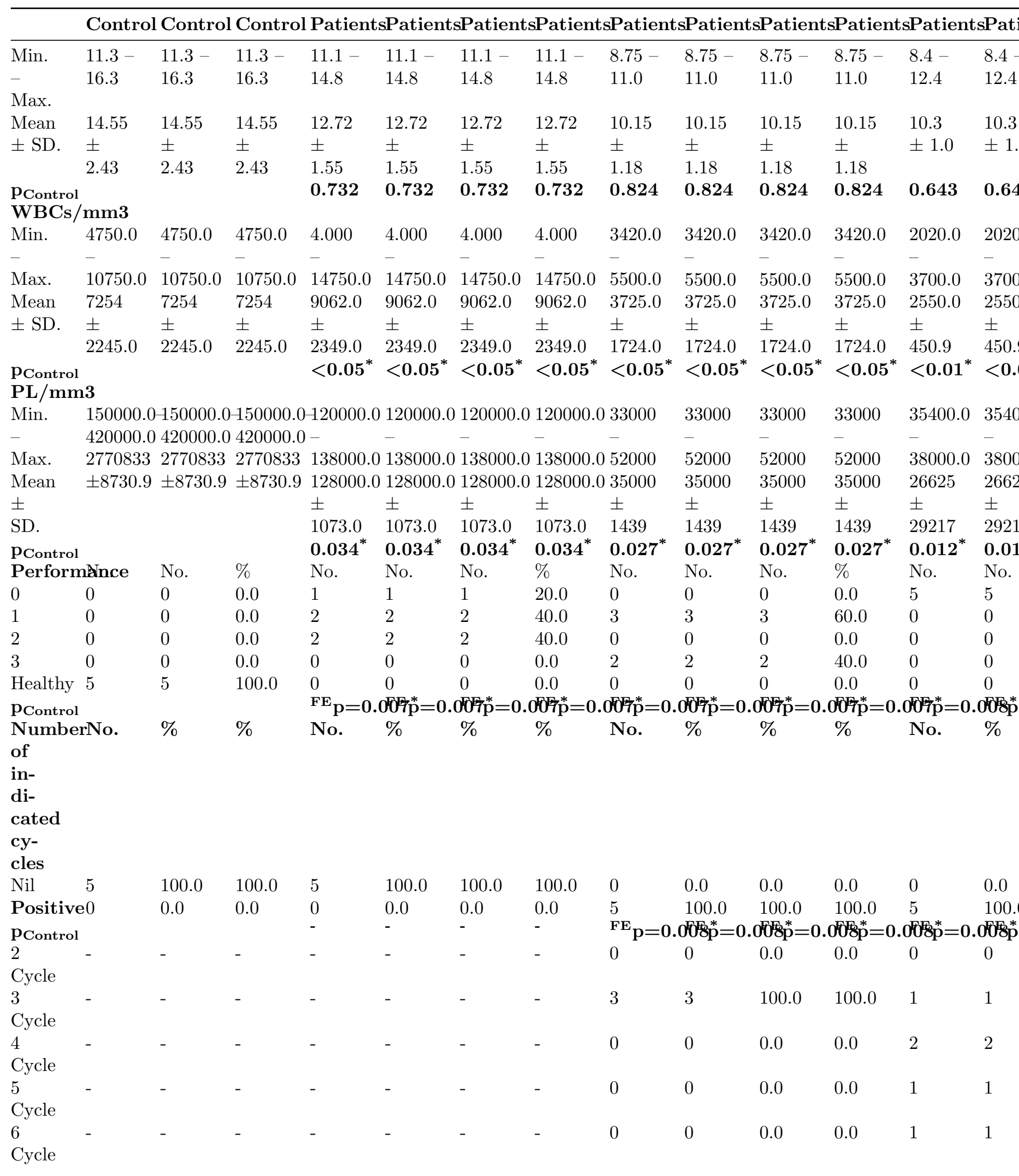




\begin{tabular}{|c|c|c|c|c|c|c|c|c|c|c|c|c|c|}
\hline & Contr & 1 Contre & Contr & Patier & sPatier & sPatier & sPatien & sPatient & sPatien & sPatien & sPatient & sPatien & sPat \\
\hline$\overline{\mathrm{MC}_{\mathrm{p}_{1}}}$ & & & & & & & & $0.008^{*}$ & $0.008^{*}$ & $0.008^{*}$ & $0.008^{*}$ & $0.008^{*}$ & 0.0 \\
\hline Viable & & & & & & & & & & & & & \\
\hline PBMCs & & & & & & & & & & & & & \\
\hline count & & & & & & & & & & & & & \\
\hline $\mathrm{ml}$ & & & & & & & & & & & & & \\
\hline af- & & & & & & & & & & & & & \\
\hline ter & & & & & & & & & & & & & \\
\hline Fi- & & & & & & & & & & & & & \\
\hline col & & & & & & & & & & & & & \\
\hline Min. & $1.27-$ & $1.27-$ & $1.27-$ & $0.53-$ & $0.53-$ & $0.53-$ & $0.53-$ & $0.47-$ & $0.47-$ & $0.47-$ & $0.47-$ & $0.48-$ & 0.48 \\
\hline- & 1.37 & 1.37 & 1.37 & 1.50 & 1.50 & 1.50 & 1.50 & 0.65 & 0.65 & 0.65 & 0.65 & 2.50 & 2.50 \\
\hline Max. & & & & & & & & & & & & & \\
\hline Mean & 1.31 & 1.31 & 1.31 & 1.01 & 1.01 & 1.01 & 1.01 & 0.60 & 0.60 & 0.60 & 0.60 & 1.17 & 1.17 \\
\hline$\pm \mathrm{SD}$ & \pm & \pm & \pm & \pm & \pm & \pm & \pm & & & & & & \pm \\
\hline & 0.05 & 0.05 & 0.05 & 0.44 & 0.44 & 0.44 & 0.44 & 0.08 & 0.08 & 0.08 & 0.08 & 0.85 & 0.85 \\
\hline $\mathbf{p}_{\text {Control }}$ & & & & 0.310 & 0.310 & 0.310 & 0.310 & $0.008^{*}$ & $0.008^{*}$ & $0.008^{*}$ & $0.008^{*}$ & 0.690 & 0.6 \\
\hline $\begin{array}{l}\text { Lymph } \\
\%\end{array}$ & 31.5 & 31.5 & 31.5 & 69.36 & 69.36 & 69.36 & 69.36 & 54.95 & 54.95 & 54.95 & 54.95 & 49.85 & 49.8 \\
\hline $\begin{array}{l}\text { Lymph } \\
\left(10^{3}\right)\end{array}$ & 2.6 & 2.6 & 2.6 & 2.84 & 2.84 & 2.84 & 2.84 & 1.48 & 1.48 & 1.48 & 1.48 & 0.77 & 0.77 \\
\hline $\begin{array}{l}\text { Mono } \\
\%\end{array}$ & 3 & 3 & 3 & 8.4 & 8.4 & 8.4 & 8.4 & 7.8 & 7.8 & 7.8 & 7.8 & 6.025 & 6.02 \\
\hline $\begin{array}{l}\text { Mono } \\
\left(10^{3}\right)\end{array}$ & 0.255 & 0.255 & 0.255 & 0.344 & 0.344 & 0.344 & 0.344 & 0.210 & 0.210 & 0.210 & 0.210 & 0.093 & 0.09 \\
\hline $\begin{array}{l}\text { Gran } \\
\%\end{array}$ & 60 & 60 & 60 & 22.64 & 22.64 & 22.64 & 22.64 & 39.025 & 39.025 & 39.025 & 39.025 & 42.313 & 42.3 \\
\hline $\begin{array}{l}\text { Gran } \\
\left(10^{3}\right)\end{array}$ & 5.1 & 5.1 & 5.1 & 0.92 & 0.92 & 0.92 & 0.92 & 1.05 & 1.05 & 1.05 & 1.05 & 0.65 & 0.65 \\
\hline
\end{tabular}

FE: Fisher Exact MC: Monte Carlo

$\mathbf{p}_{\text {Control }}$ : p value for comparing between control and each other group

$\mathrm{p}_{1}$ : $\mathrm{p}$ value for comparing between during and

after

*: Statistically significant at p [?] 0.05 